\title{
High-Energy Collision-Induced Dissociation of Ceramide Ions from Permethylated Glycosphingolipids
}

\author{
D. D. Ju, G. J. Wei, and G. R. Her \\ Department of Chemistry, National Taiwan University, Taipei, Taiwan, Republic of China
}

\begin{abstract}
Ceramide fragments from permethylated glycosphingolipids (GSLs) were studied by highenergy collision-induced dissociation (CID). In comparison with ceramide fragments of underivatized GSLs, many more product ions including charge-remote fragment ions were observed. These ions provided detailed structural information on the ceramides. The relative intensity and the mass interval between the $L$ and $M$ ions were used to assign the position of the double bond. The position of the hydroxyl group was assigned with the $\mathbf{L n}$ and $\mathrm{K}$ ions. Because the ceramide fragments and not the pseudomolecular ions were selected as the precursor ions, the size of GSLs had little effect on the quality of the product ion spectra. The sensitivity of this approach was in the range of picomoles. (J Am Soc Mass Spectrom 1994, 5, 558-563)
\end{abstract}

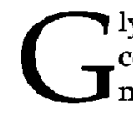
lycosphingolipids (CSLs) are cell membrane components. There are two different structural moieties in GSLs: a ceramide and a carbohydrate residue. The ceramide consists of the long chain base (sphingoid) substituted at the amino group by a fatty acid. The growing interest in the diverse biological functions of GSLs as cell surface anligens, cell-cell recognition markers, and receptors for a variety of signal molecules has stimulated the search for new analytical techniques for their structural analysis.

Mass spectrometry, a highly sensitive technique, has been a key tool for the structural elucidation of GSLs. The classical approach involved the conversion of GSLs to methyl, acetyl, or trimethylsilyl derivatives before electron ionization (EI) or chemical ionization (CI) mass spectrometric analysis [1-4]. I his approach proved to be very useful to the structural analysis of glycans and ceramide. Since its development in the early 1980s, fast-atom bombardment (FAB) has quickly become the method of choice for the analysis of polar, nonvolatile compounds such as peptides, oligosaccharides, and GSLs. FAB, both in the positive and negative ion mode, has been widely used in the structural analysis of derivatized and underivatized GSLs [5-9].

Mainly due to the many interfering matrix ions and the lack of structurally important fragment ions, recent works focus on the use of FAB in combination with collision-induced dissociation (CID) in the structural analysis of GSLs and ceramides [10-17]. Costello and

Address reprint requests to G. R. Her, Department of Chemistry. National Taiwan University, Taipei, Taiwan, Republic of China. co-workers have demonstrated the utility of using FAB in combination with four-sector tandem mass spectrometry to elucidate the structure of GSLs [12-14]. High-energy CID of the ccramide fragments from underivatized GSLs provides information on the mass of the sphingoid and $\mathrm{N}$-acyl residue [14]. However, the location of other substituents such as double bonds and hydroxyl groups in the sphingoid and $\mathrm{N}$-acyl chain cannot be determined. For intact ceramides, in addition to the much beller sensilivity under FAB conditions, the procedure of amide reduction, hydroboration and oxidation followed by high energy CID of the $(\mathrm{M}+\mathrm{H})^{+}$ion provides the complete structural information of the ceramide including the position of the double bonds $[13,14]$. High energy CID of metal adduct ions of GSLs and ceramides has been studied extensively by Ann and Adams [15-17]. For the cationized (especially lithium) ceramides and small GSLs, the CID spectra provide information on the length of the long chain base and fatty acid, as well as the location of the double bond and hydroxyl group in the $\mathrm{N}$-acyl chain. However, this method has not been applied to GSLs with more than two sugar residues.

A low-energy CID study of ceramide ions from permethylated GSLs has been reported [18]. The product ions provide information about the length of the fatty acid and long chain base. However, ions related to the position of the double bonds and hydroxyl groups were not detected. In this article, we explore the potential of using high energy CID to study the structure of the permethylated ceramide fragments from GSLs with one-seven sugar residues. 


\section{Experimental}

\section{Materials and Methods}

Glucocerebrosides from human (Gaucher's) spleen, type 1 and type 2 galactocerebrosides from bovine brain, N-palmitoyl-, N-stearoyl-, N-lignoceroyl-dihydrolactocerebroside, N-palmitoyl-, N-oleoyl-, Nstearoyl-, and N-nervonoyl-cerebrosides, Gangliosides GM1, GD1a, GT1b were all purchased from Sigma Inc. (St Louis, MO). Helium and xenon were purchased from San-Fu Co. (Hsinchu, Taiwan). GSLs were permethylated as described by Ciucanu and Kerek [19], and adapted for glycolipids by Larson et al. [20]. Briefly, a sample was put into a screw-top glass tube and subsequently dissolved in $200 \mu \mathrm{L}$ of anhydrous dimethyl sulfoxide which contained finely powdered sodium hydroxyde. The solution was vortexed for 4 min at room temperature. Ten microliters of methyl iodide (trideuteromethyl iodide for deuteromethylation) was added and the solution was sonicated at room temperature for an additional hour. Dichloromethane $(1 \mathrm{~mL})$ and water $(1 \mathrm{~mL})$ were added to the reaction mixture. After vortex and centrifugation, the dichloromethane phase was washed three times with 2 $\mathrm{mL}$ of water.

\section{Mass Spectrometry}

All spectra were recorded with a Jeol SX-102A (JEOL, Japan) double focusing mass spectrometer of reversed geometry. In FAB/MS, the FAB gun was operated at 6 $\mathrm{kV}$ using xenon as the ionizing gas. One microliter of a sample solution was mixed with $1 \mu \mathrm{L}$ of matrix (3nitrobenzyl alcohol) on the FAB probe tip for FAB and $\mathrm{FAB} / \mathrm{CID}$ analysis.

Mass spectra from constant $B / E$ (product) linked scans and mass-analyzed ion kinetic energy mass spectra were acquired at a scan rate of $20 \mathrm{~s} / \mathrm{scan}$. Helium was used as the collision gas; the pressure of helium was adjusted to reduce the ion beam to $50 \%$ of its initial value. The mass scale in the linked scan mode was calibrated with a mixture of alkali metal halides [21].

\section{Results and Discussion}

The formation of permethylated derivatives for the analysis of glycolipids by positive ion $F A B$ is a wellestablished strategy. Under the conditions of $\mathrm{FAB}$, permethylated GSLs showed much higher sensitivity than their underivatized analogs [13]. Because of the chemical noise and/or the lack of structurally important fragment ions in FAB mass spectra, CID was chosen to elucidate the detail structure of the GSLs. When the FAB-generated $\mathrm{MH}^{+}$ions were studied by high-energy $C I D$, the spectra were primarily composed of fragment ions resulting from cleavage of the glycosidic linkages with minimal fragmentation in the ce- ramide portion of the molecule. The lack of sphingoidand $\mathrm{N}$-acyl chain-related product ions suggested the need for acquiring product ion mass spectra of the ceramide fragments. Ceramide ions ( $Z$ type ion, using the nomenclature proposed by Costello et al. [14]) were often observed as one of the major fragments under the condition of positive ion FAB [22]. When the ceramide fragments of permethylated GSLs were studied by high-energy $\mathrm{CID}$, the spectra were very much different from the product ion spectra of underivatized ceramide fragments [14] in that many more product ions, including the charge-remote losses of $\mathrm{C}_{\mathrm{n}} \mathrm{H}_{2 \mathrm{n}}$ and $\mathrm{H}_{2}$ (or $\mathrm{C}_{\mathrm{n}} \mathrm{H}_{2 \mathrm{n}+2}$ ) from the $\mathrm{N}$-acyl chain, were observed. The nomenclature and sites of cleavage of the major product ions are shown in Scheme I. The nomenclature used is not all the same as those proposed previously $[14,15-17]$ because some of the product ions have not been reported before and the compounds studied are permethylated GSLs rather than underivatized GSLs. Fn ( $\mathbf{n}=1,2,3 \ldots)$ is used for ions containing the $\mathrm{N}$-acyl chain, whereas $\mathrm{Ln}$ is used for ions containing the long chain base. The nomenclature proposed by Adams [15-17] was adopted for ions resulting from the charge remote losses of alkyl groups from fatty acyl chains ( $K, L$, and $M$ ions). The product ion mass spectra of the ceramide fragments from the permethylated N-palmitoyl-, N-stearoyl-, and N-lignoceroyl-dihydrolactocerebrosides are shown in Figure 1. The L1 $(m / z 280)$ and $\mathbf{F 1}$ ions $(m / z 270,298,382)$ indicated the chain length of the long chain base and fatty acid, respectively. These assignments were further supported by the $\mathbf{L 2}(\mathrm{m} / z$ 310/312) and $\mathbf{F} 2(\mathrm{~m} / z$ $294,322,406$ ) ions. The series of ions due to the loss of $14 n+2$ were believed to be similar to those observed by Ann and Adams [15-17] in the CID of metal cationized ceramides. Most likely due to the conjugation (Scheme IIa), the $\mathbf{K}$ ion $(m / z$ 366) has a higher abundance than the analogous higher mass ions [16]. The F3' ions $(m / z 324,352,436)$ were assigned tentatively as the loss of $\mathrm{C}_{15} \mathrm{H}_{30}$ and $\mathrm{CH}_{4}$ from the sphinganine. In the study of trideuteromethylated N-stearoyl-dihydrolactocerebroside, the $\mathrm{FJ}^{\prime}$ ion was observed at $m / z$ 355 (elimination of $\mathrm{C}_{15} \mathrm{H}_{30}$ and $\mathrm{CD}_{3} \mathrm{H}$ ) instead of 358

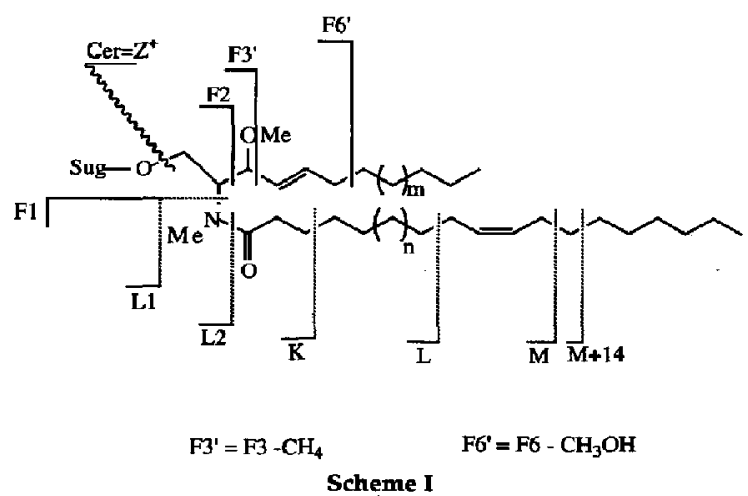




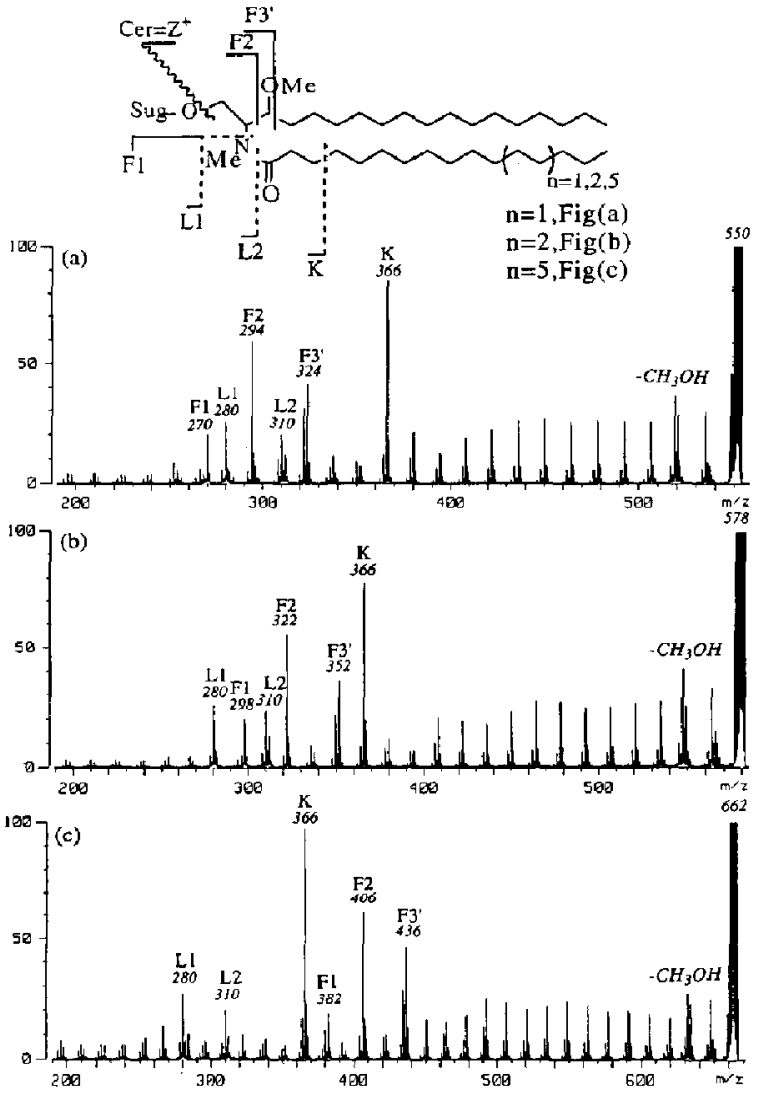

Figure 1. The prodtuct ion spectra, obtained with linked scanning at constant $B / E$, of the ceramide fragments from the permethylated (a) N-palmitoyl-dihydro-lactocerebrosides, $m / z$ 550, (b) N-stearoyl-dihydro-lactocerebrosides, $m / z$ 578, and (c) $\mathrm{N}$ lignoceroyl-dihydro-lactocerebrosides, $m / z$ 662. In all spectra, the masses of the precursor and product ions are shown as norminal masses (e.g., $m / z 550$ is actually 550.7).

$\mathbf{a}$

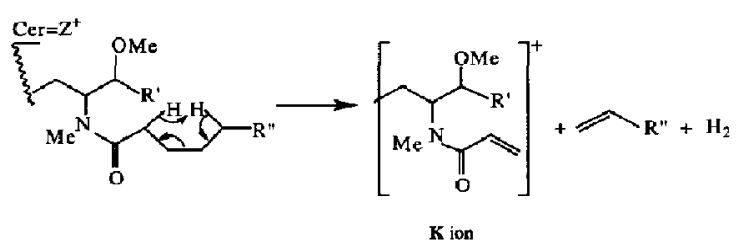

b

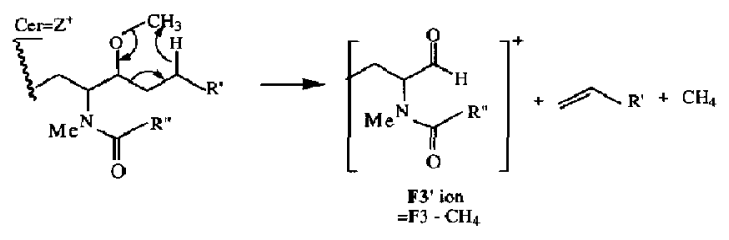

Scheme II (elimination of $\mathrm{C}_{15} \mathrm{H}_{30}$ and $\mathrm{CH}_{4}$ ). These data are consistent with the postulation of cleavage of the methyl group from the C(3) methoxy group of the sphinganine (Scheme IIb).

GSLs containing an unsaturated long chain base, such as (4E)-sphingenine, are more common. The product ion mass spectra of the permethylated N-palmitoyland $\mathrm{N}$-stearoyl-cerebrosides are shown in Figure 2. Unlike ceramides with a saturated long chain base, the F1 (acylamide) ion was not observed (Figure 2), which indicated the significant influence of the double bond in the long chain base on the formation of the F1 ion. The $\mathrm{N}$-acyl chains in Figure $1 \mathrm{a}, \mathrm{b}$ are the same as in Figure 2. Thus, the ions containing fatty acid (F2, F3') had the same mass-to-charge ratio values. Because of the presence of a double bond in the long chain base, the ions containing the sphingoid (L1, L2, K) were, as expected, two mass units less than the aralogous ions observed in Figure 1. In addition to the F2, F3', L1, L2, and $\mathbf{K}$ ions, an ion ( $\mathbf{F}^{\prime}$ ) indicating the position of the double bond $\left(\mathrm{C}_{4}-\mathrm{C}_{5}\right)$ in the sphingoid was also observed (Figure 2). This ion corresponds to the elimination of $\mathrm{C}_{n} \mathrm{H}_{2 n}$ and a methanol molecule from the ceramide ion to form a conjugated diene (Scheme III). The elimination of the $C(3)$ methoxy group in the long chain base was supported, in part, by the observation of the $m / z 379$ ion (elimination of $\mathrm{C}_{12} \mathrm{H}_{24}$ and $\mathrm{CD}_{3} \mathrm{OH}$ ) for the trideuteromethylated $\mathrm{N}$-stearoylcerebroside ( $m / z 376$ for permethylated $N$-stearoylcerebroside).

The observation of the $[Z-(14 n+2)]$ ions presents the possibility to detect the location of the double bonds or other substituents in the sphingoid or $\mathrm{N}$-acyl chains. The product ion mass spectra of ceramide fragments with a double bond in the fatty acyl chain (N-oleoyl- and N-nervonoyl-cerebrosides) are shown in Figure 3. Similar to the CID data reported by Ann and Adams [16] for the $(\mathrm{M}+\mathrm{Li})^{+}$ions, ions between the $\mathbf{L}$ and $\mathbf{M}$ ions are less abundant than the $\mathbf{L}$ and $\mathbf{M}$ ions. In addition to the reduction in ion abundance, the mass interval between the $L$ and $M$ ions is $54 \mathrm{u}\left(\mathrm{C}_{4} \mathrm{H}_{6}\right)$ rather than $56 \mathrm{u}\left(\mathrm{C}_{4} \mathrm{H}_{8}\right)$. The ion $14 \mathrm{u}$ higher than the $M$ ion $(M+14$ ion) has higher abundance than the analogous higher mass ions, which can be rationalized due to the formation of a stable conjugated diene (Scheme IV). This approach for double bond assignment was tested with type 2 galactocerebroside. The $\mathbf{Z}$ ion from the permethylated type 2 galactucerebrosidt was observed at $m / z$ 658. The product ion mass spectrum of the 658 ion is very similar to Figure $3 \mathrm{~b}$; thus, the fatty acid in the type 2 galactocerebroside was identified to be a C24:1 fatty acid with a double bond in the $\mathrm{C}(15)-\mathrm{C}(16)$ position (nervonic acid). The hydroxyl group in the $N$-acyl chain could alsu be assigned with this method. The $Z$ ion from the permethylated type 1 galactocerebroside was observed at $m / z$ 690. In comparison with the product ion mass spectrum of N-nervonoyl-cerebroside (Figure $3 \mathrm{~b}$ ), the same mass-to-charge ratio values for the $\operatorname{Ln}$ ions $(m / z 278$, $308 / 310$ ) suggests that the hydroxyl group is in the 


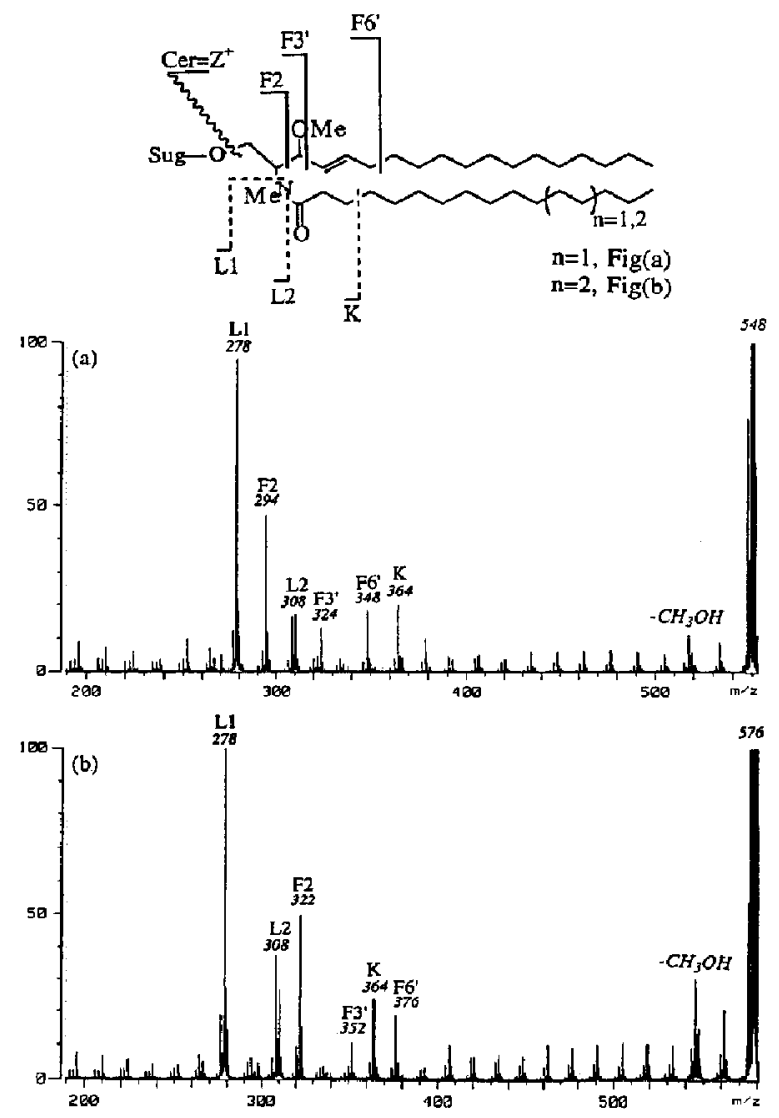

Figure 2. The product ion spectra, obtained with linked scanning at constant $\mathrm{B} / \mathrm{E}$, of the ceramide fragments from the permethylated (a) $\mathrm{N}$-palmitoyl-cerebroside, $m / z$ 548, (b) $\mathrm{N}$ stearoylcerebrosides, $m / z 576$.

fatty acid rather than the long chain base. The hydroxyl group was determined to be in the $\alpha$ position of the lignoceric acid because the $K$ ion was observed at $m / z 394$ which is 30 mass units (-OCH3 versus $-\mathrm{H}$ ) higher than the $\mathbf{K}$ ion observed in Figures 2 and 3.

A product ion spectrum obtained using a linked scan of constant B/E shows good product ion resolution; however, the resolution in the selection of the precursor ion is not adequate for ceramides with very similar mass-to-charge ratio values. In the analysis of

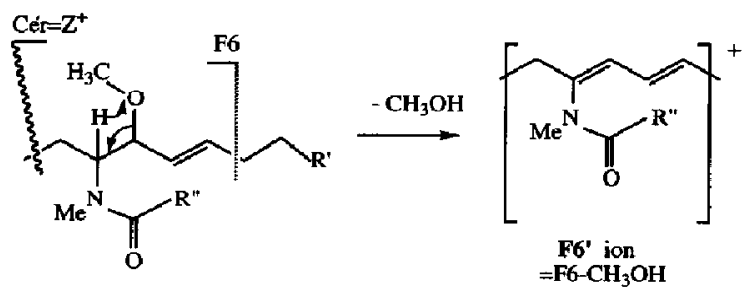

Scheme III
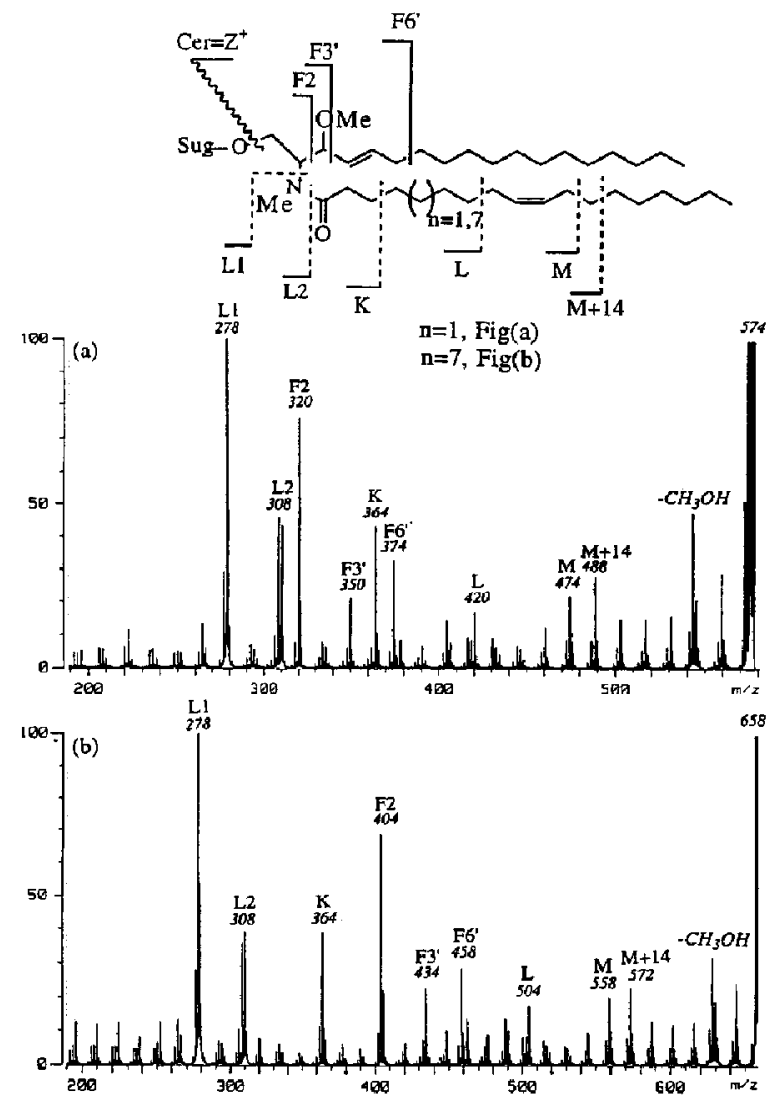

Figure 3. The product ion spectra, obtained with linked scanning at constant $B / E$, of the ceramide fragments from the permethylated (a) N-oleoyl-cerebroside, $m / z$ 574, (b) N-nervonoylcerebroside, $m / z 658$

glucocerebrosides, a mixture with microheterogeneity in the ceramide, four different ceramide ions $(\mathrm{m} / \mathrm{z}$ $660,658,632,548)$ were observed. Based on their linked scan at constant $B / E$ mass spectra (not presented), the fatty acids in the $m / z 632$ and 548 ions were assigned
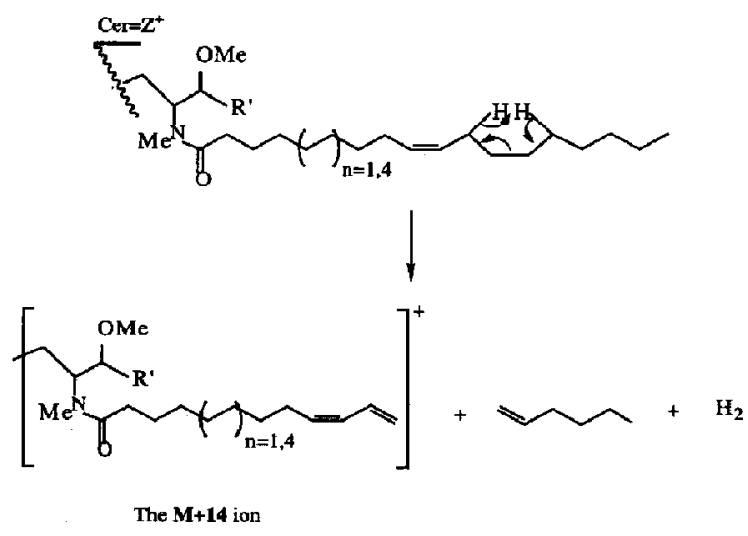

Scheme IV 
as behenic acid and palmitic acid, respectively. Because linked scan at constant $B$ /E does not provide adequate resolution for the differentiation of $m / z 66 \mathrm{U}$ and 658 ions, mass selection followed by ion kinetic energy analysis was used to acquire the product ion spectra of the $m / z 660$ and 658 ions (Figure 4). The $L n$ ions $(m / z 278,308)$ and $\mathbf{K}$ ion $(m / z 364)$ in Figure $4 a$ and $b$ were the same as those observed in Figure 3b; therefore, the long chain base was assigned as a sphing-4-ene residue. The F2, $\mathbf{F}^{\prime}$, and $\mathbf{F}^{\prime}$ ions at $m / z$ $406,436,460$ suggested that the $\mathrm{N}$-acyl chain of the $m / z 660$ ion was a lignoceric acid residue (Figure $4 b$ ). The fatty acid of the $m / z 658$ ion (Figure 4a) was determined to be a $C_{24.1}$ acid because the Fn ions $(\mathrm{m} / \mathrm{z}$ $404,434,458$ ) were two mass units less than the analogous ions observed in Figure $4 \mathrm{~b}$. Based on the $\mathrm{L}$ and $\mathbf{M}$ ions (Figure 4a), the double bond in this C24:1 acid was determined to be in the $\mathrm{C}(15)-\mathrm{C}(16)$ position (nervonic acid). In linked scan at constant $\mathrm{B} / \mathrm{E}$ mass
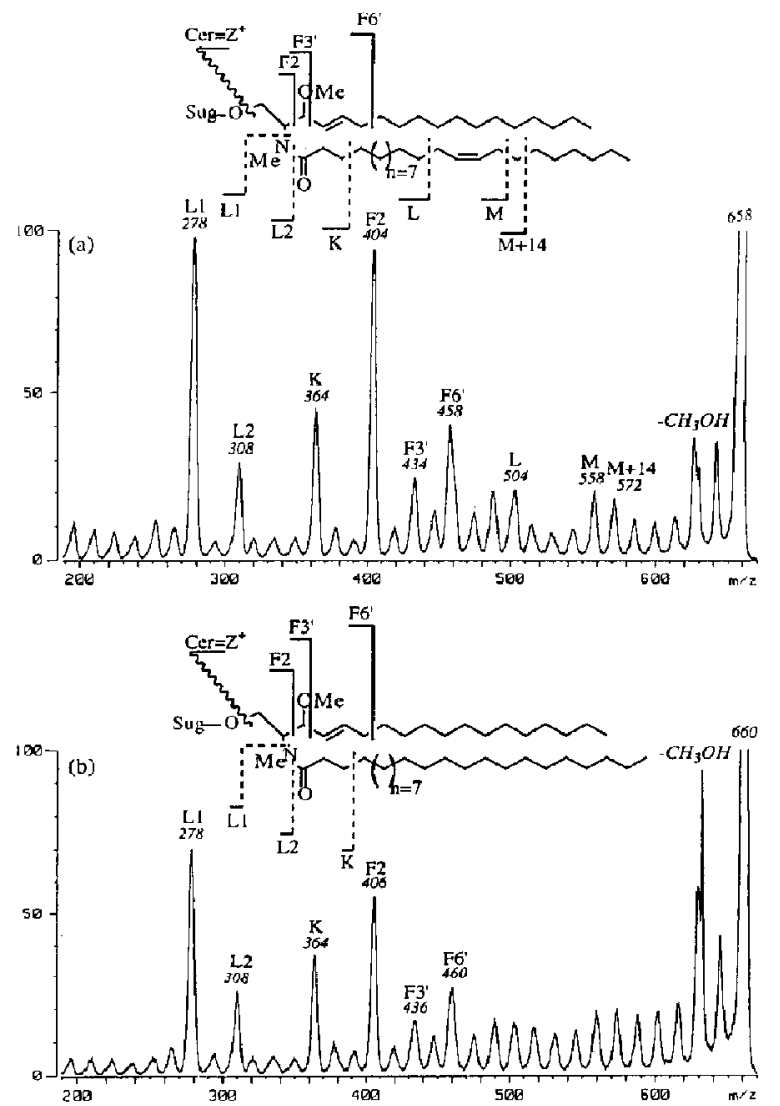

Figure 4. The product ion spectra, obtained with mass selection followed by ion kinetic energy analysis of the ceramide fragments from the permethylated glucocerebrosides. (a) The $m / z$ 658 precursor ion. (b) The $m / z 660$ precursor ion. Glucocerebrosides were isolated from human (Gaucher's) spleen. Four different GSLs were detected in this mixture; two of the four product ion spectra are shown in this figure. spectra, the position of the double bond is determined according to the abundance and the mass separation (54 $u$ ) of the $L$ and $M$ ions. Because of the poor product ion resolution in the MIKES, there is some uncertainty in the mass assignment of the $\mathbf{L}$ and $\mathbf{M}$ ions. Therefore, the position of the double bond was assigned mainly based on the abundance of the $\mathbf{L}$ and $\mathbf{M}$ ions. In samples where two or more ceramide fragments have very similar mass and the difference in relative intensity is less obvious, a four-sector tandem mass spectrometer would be needed to make a clear assignment.

It is in the analysis of GSLs, rather than intact ceramides, that this approach showed a clear advantage over the published methods. Due to the size of the glycan, many GSLs have molecular weights much larger than ceramides and cerebrosides. Because the ceramide fragments rather than the $\mathrm{MH}^{+}$ions were selected as the precursor ions in this approach, the masses of the precursor ions remain in the range of $500-700$ u. This represents a much more favorable situation than the methods with the selection of the $(\mathrm{M}+\mathrm{H})^{+},(\mathrm{M}-\mathrm{H})^{-}$, or $(\mathrm{M}+\mathrm{Li})^{+}$as the precursor ions because the quality of the product ion spectra often becomes rapidly worse with increasing precursor ion mass. For example, the method of metal ion adduction provides an excellent approach for ceramides and small GSLs [15-17]. To our knowledge, this method has not been applied to GSLs with more than two sugar residues. This is, at least in part, due to the fact that the product ion spectra of large GSLs do not have the quality of those of ceramides and small GSLs.

Sensitivity is important in the analysis of GSLs because GSLs are often isolated in small amounts. The detection limit of this method compares favorably with published methods. In the analysis of a GSL with two sugar residues, N-palmitoyl-dihydrolactocerebroside, 5 pmol of the permethylated sample (under the assumption of $100 \%$ yield in permethylation) produced the product ion mass spectrum shown in Figure 5. For a much larger GSL, GT1b, the FAB mass spectrum was characterized with ceramide ions $(m / z 576,604)$ and carbohydrate containing ions as shown in Figure 6a. The product ion mass spectrum of the $m / z 604$ ion trom 46 pmol of the permethylated GTlb is shown in Figure $6 \mathrm{~b}$. Since there are two different ceramide fragments $(m / z 576,604)$ with relative intensity of 2:3 (Figure 6a), the spectrum in Figure 6b represents approximately $28 \mathrm{pmol}$ of GT1b molecules with eicosasphingosine as the long chain base. Since the molecular weight of permethylated GT1b is in the range of $2500 \mathrm{u}$ and a $6-\mathrm{keV}$ Xe gun is used in this analysis, the sensitivity might be further improved with a higher energy $\mathrm{Cs}^{+}$ion gun.

\section{Conclusions}

High-energy CID of the ceramide fragments from underivatized GSLs provides little information on the 


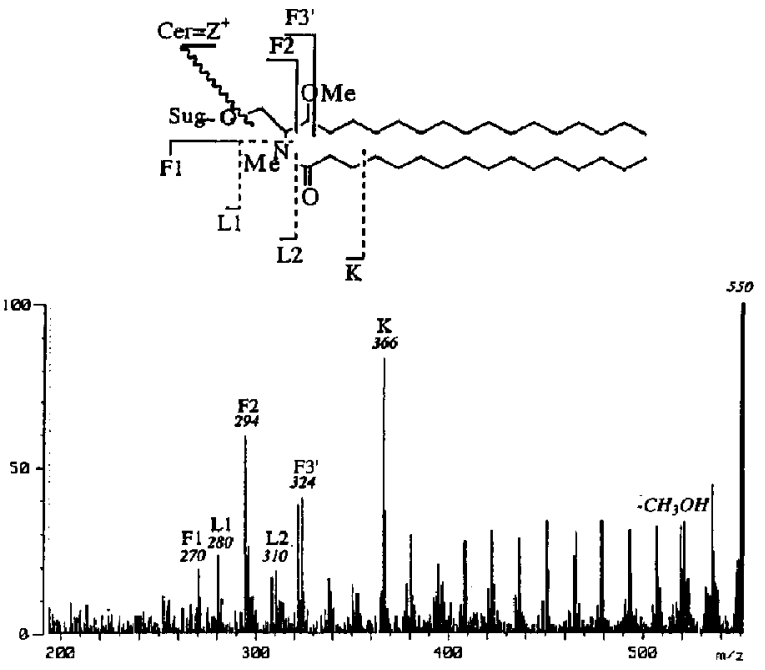

Figure 5. The product ion spectra, obtained with linked scanning at constant $B / E$, of 5 pmol of permethylated N-palmitoyldihydro-lactocerebroside.

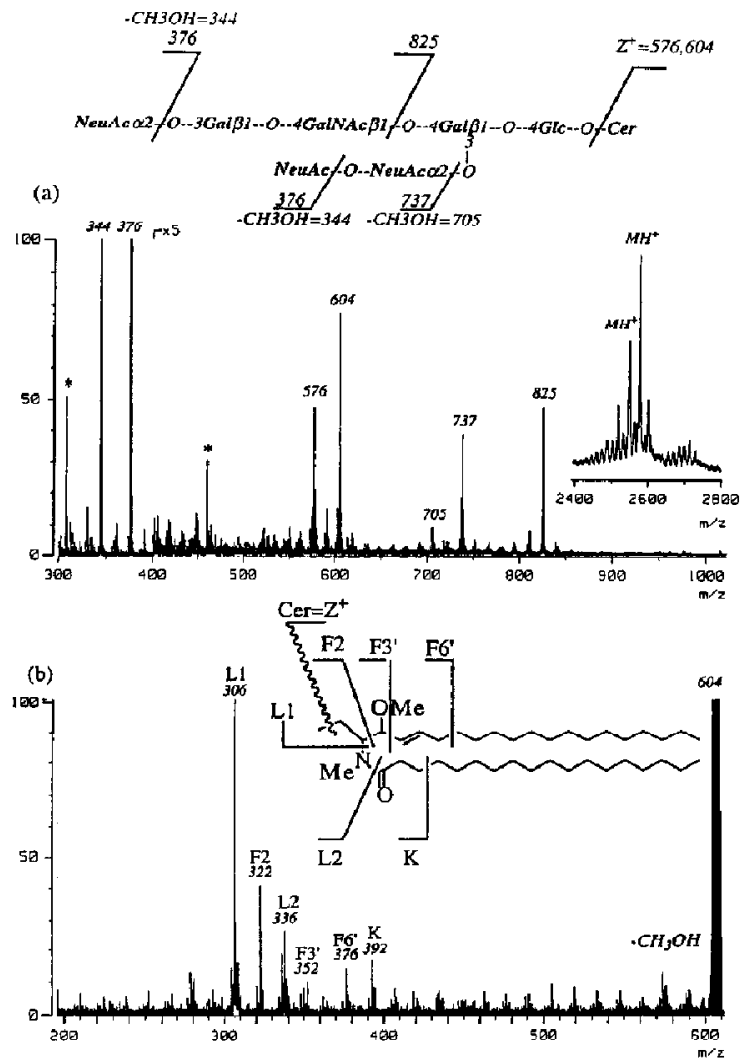

Figure 6. (a) The FAB mass spectrum of permethylated GT1b. The peaks marked with * are matrix peaks of 3-nitrobenzyl alcohol. (b) The product ion mass spectrum of the ceramide fragment at $m / z 604$ from $46 \mathrm{pmol}$ of the permethylated GT1b. The spectrum corresponds to approximately $28 \mathrm{pmol}$ of GT1b molecules with eicosasphingosine as the long chain base. location of substituents such as double bonds and hydroxyl groups in the sphingoid and $\mathrm{N}$-acyl chain [14]. We have shown that permethylation, a very simple procedure, can overcome this problem. Upon high-energy CID, the product ion mass spectra of the ceramide fragments provide detailed information regarding the structure of the ceramides. Since the ceramide fragments instead of $\mathrm{MH}^{+}$ions are chosen as the precursor ion, the molecular weight of GSLs has no effect on the mass of the precursor ions and the quality of the product ion spectrum can be preserved. The sensitivity of this approach makes possible the analysis of large GSLs in the range of picomoles.

\section{Acknowledgment}

Financial support from the National Science Council of the Republic of China is greatly appreciated.

\section{References}

1. Karlssion, K.-A. FEBS Lett. 1973, 32, 317-320.

2. Teneberg, S.; Pimloot, W.; Karlsson, K.-A. In Biological Mass Spectrometry; A. L. Burlingame and J. A. McCloskey, Ed.; John Wiley: New York, 1990; pp. 477-490.

3. Ariga, T.; Yu, R. K.; Suzuki, M.; Ando, S.; Miyatake, T. I. Lipid Res. 1982, 23, 437-442.

4. Carr, S. A.; Reinhold, V. N. Biomed. Mass Spectrom. 1984, 11, 633-641.

5. Kushi, Y.; Handa, S. J. Biochem. 1982, 91, 923-931.

6. Hemling, M. E.; Yu, R. K.; Sedgwick, R. D.; Rinehart, K. L., Jr. Biochemistry. 1984, 23, 5706-5713.

7. Arita, M.; Iwamori, M.; Higuchi, I'; Nagai, Y. J. Biochem. 1984, 95, 971-981.

8. Iwamori, M.; Ohashi, Y.; Nagai, Y. In Mass Spectrometry in the Health and Life Sciences; $\Lambda$. L. Burlingame and N. Castagholi, Jr., Eds.; 1985, pp. 379-398.

9. Pahlsson, P.; Nilsson, B. Anal. Biochem. 1988, 168, 115-120.

10. Kushi, Y.; Rokukawa, C.; Handa, S. Anal. Biochem. 1988, '775, 167-176.

11. Ohashi, Y.; Iwamori, M.; Ogawa, T.; Nagai, Y. Biochemistry 1987, 26, 3990-3995.

12. Domon, B.; Costello, C. E. Biochemistry 1988, 27, 1534-1543.

13. Domon, B.; Vath, J. E.; Costello, C. E. Anal. Biochem. 1990, 184, 151-164.

14. Costello, C. E.; Vath, J. E. In Methods in Lnzymology; McCloskey, J. A., Ed.; Academic Press: San Diego, CA, 1990; Vol. 193, pp. 738-768.

15. Ann. O.: Adams, J. J. Am. Soc. Mass Spectrom. 1992, 3, 260-263.

16. Ann, Q.; Adams, J. Anal. Chem. 1993, 65, 7-13.

17. Adams, J.; Ann, Q. Mass Spectrom. Rev. 1993, 12, 51-85.

18. Duh, J. S.; Her, G. R. Biological Mass Spectrometry 1992, 21, 391-396.

19. Ciucanu, I.; Kerek, F. Carbohydr. Res. 1984, 131, 209-217.

20. Larson, G.j Karlssin, H.; Hanlssun, G. C.; Pindutt, W. Curbuhydr. Res. 1987, 161, 281-290.

21. Sato, K.; Asada, T.; Ishihara, M.; Kunihiro, F.; Kammei, , Y.; Kubota, E; Costello, C. E; Martin, S. A.; Scoble, H. A.; Biemann, K Anal. Chem. 1987, 59, 1652-1659.

22. Guo, N.; Her, G. R.; Reinhold, V.; Brennan, M. J.; Siraganian, R. P.; Ginsburg, V. J. Biol. Chem. 1989, 264, 13267-13272. 